ASTHMA

\title{
Effects of inhaled fluticasone on angiogenesis and vascular endothelial growth factor in asthma
}

\author{
B N Feltis, D Wignarajah, D W Reid, C Ward, R Harding, E H Walters
}

Thorax 2007;62:314-319. doi: 10.1136/thx.2006.069229

See end of article for authors' affiliations

......................

Correspondence to: Dr B Feltis, Tasmanian School of Medicine, 43 Collins St, Hobart,

Tasmania, Australia 7001; bryce.feltis@csiro.au

Received 25 July 2006

Accepted 12 October 2006

Published Online First

13 November 2006
Background: Subepithelial hypervascularity and angiogenesis in the airways are part of structural remodelling of the airway wall in asthma, but the effects of inhaled corticosteroids (ICS) on these have not been explored. Increased vascularity in asthma may contribute to a number of functional abnormalities. A study was undertaken to explore angiogenic modulation by ICS and its likely regulation via vascular endothelial growth factor (VEGF), its receptors and the angiopoietins.

Methods: A placebo-controlled intervention study with ICS in asthma was performed, examining vascularity, VEGF, its receptors (VEGFR1 and VEGFR2), and angiopoietin-1 (Ang 1) to assess which of these factors were changed in the asthmatic airways after ICS treatment. Airway wall biopsy specimens, lavage fluid and cells were obtained from 35 patients with mild asthma randomised to receive ICS or placebo for 3 months, after which bronchoscopic examination and sample collection were repeated. Immunohistochemistry and image analysis were used to obtain quantitative measures of vessels, angiogenic sprouts, VEGF, VEGFR1, VEGFR2 and Angl staining in airway biopsy specimens. ELISA was used to assess VEGF concentrations in the lavage fluid.

Results: Vessel, VEGF and sprout staining were decreased after 3 months of ICS treatment. VEGF levels remained unchanged. VEGF receptors and Angl staining were not reduced after treatment.

Conclusions: The findings of this study support an effect of ICS in downregulating angiogenic remodelling in the airways in asthma, associated with decreasing VEGF activity within the airway wall. The environment of the airways after treatment with ICS, with changes in the balance between VEGF, its receptors, Angl and sprouts, appears to be less angiogenic than in untreated asthma.
A sthma is characterised by variable airflow obstruction, airway hyper-responsiveness, chronic airway inflammation and airway wall structural remodelling. Inhaled corticosteroids (ICS) are the most effective treatment currently available for inducing symptomatic control, and have become the mainstay of modern asthma treatment. ${ }^{1}$ ICS suppress the chronic inflammation of asthma and improve airway hyperresponsiveness and lung function. ${ }^{23}$ Their main advantage is high efficacy combined with few systemic side effects compared with oral steroids such as prednisolone. ${ }^{4}$ The effects of current treatment regimens on structural airway changes in asthma are less well established. We have recently shown beneficial effects on subepithelial reticular basement membrane scarring, although this required more protracted treatment compared with effects on inflammation.

Airway remodelling is believed to occur in concert with, or as a result of, ongoing airway damage resulting from airway inflammation. Bronchial hyper-responsiveness (BHR) is characteristic of asthmatic airways and seems to be related equally to airways inflammation and remodelling. ${ }^{5}$ Anti-inflammatory corticosteroids reverse BHR, and this seems to be due to effects on both airway inflammation and remodelling. However, the temporal changes in the pathological domain of asthma may be different, although there are as yet few data at this detailed level.

Previous work from our group and laboratory has shown that the airways of steroid-naive asthmatics have more vessels and a greater percentage area of vasculature in the lamina propria immediately below the epithelium than normal control subjects. ${ }^{6}$ Hypervascularity of the subepithelial lamina propria in asthma airways is beginning to attract increased attention, with several recent studies having investigated its contribution to airway pathology in asthma. ${ }^{78}$ There are a small number of studies on the effects of treatment with ICS. ${ }^{9}$ In cross-sectional studies the increased vascular indices in asthma have been related to functional changes such as lung function, BHR and dose of ICS. ${ }^{1011}$

Vascular endothelial growth factor (VEGF) is a multifunctional growth factor with potent actions on vascular endothelial cells, inducing mitogenesis, increased endothelial permeability and enhanced cell migration. In asthma, VEGF has been implicated in airway vascular remodelling either by increasing vascular permeability and tissue oedema and/or stimulation of new blood vessel growth. ${ }^{9}$ Both effects may lead to increased airway wall thickness and airflow limitation, especially in the presence of enhanced contraction of the surrounding muscle layer. ${ }^{12}$ Angiopoietin-1 (Angl) is another angiogenic growth factor which acts both in series with and subsequent to VEGF stimulation. It helps to maintain and stabilise mature vessels by promoting interactions between endothelial cells and surrounding support cells such as pericytes. ${ }^{13}{ }^{14}$ Thus, VEGF and Angl are likely to complement each other in angiogenic processes within the airways of asthmatics.

In the present study we have continued our examination of vascular "sprouts" which we reported recently. ${ }^{15}$ We have suggested that these cystic structures in the vascular wall of airway vessels may be newly forming vessels or, at least, indicators of enhanced remodelling processes. We previously reported differences between patients with asthma and controls

Abbreviations: Angl, angiopoietin-1; BAL, bronchoalveolar lavage; BHR, bronchial hyper-responsiveness; FP, fluticasone propionate; GMA, glycol methacrylate; ICS, inhaled corticosteroids; VEGF, vascular endothelial growth factor; VEGFR1, VEGFR2, vascular endothelial growth factor receptors R1 and R2 


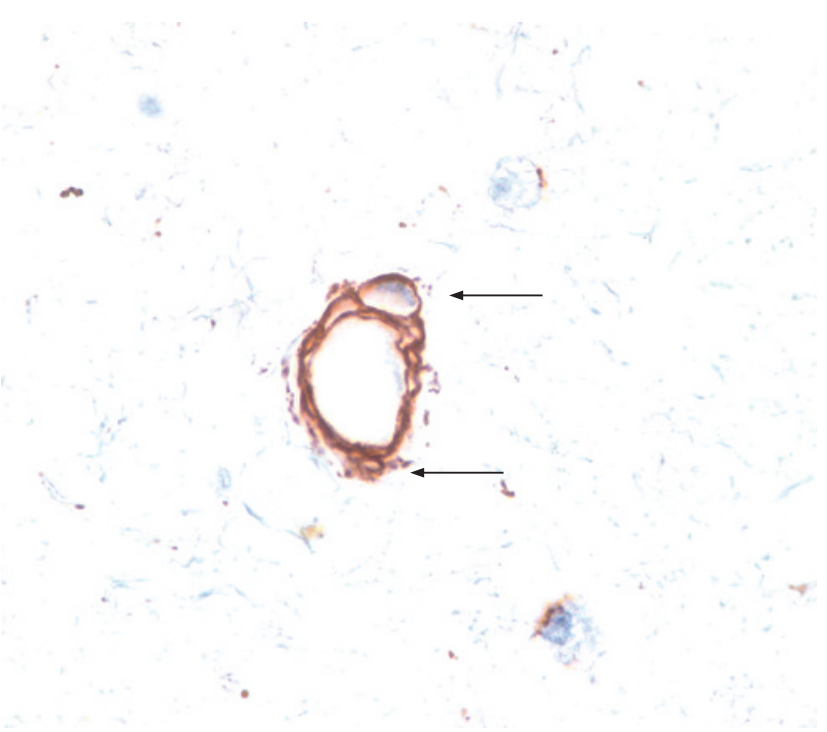

Figure 1 Immunohistochemical staining of vascular "sprouts". Endobronchial biopsy specimens obtained from asthmatic subjects were immunostained with collagen type IV antibody to highlight the endothelial basement membrane of blood vessels located within the lamina propria. "Sprouts" were defined as the unusual vessel-like spaces located within the lining of the vessel wall (arrows).

in these structures, and have now examined the effects of ICS on these sprouts.

The effect of ICS on airway mucosal vascularity in patients with asthma has not been fully established. No studies to date have examined the influence of ICS on VEGF or Angl or their potential contributions to microvascular remodelling in asthma. In this study we aimed to determine whether high dose ICS (750 $\mu$ g fluticasone propionate (FP) twice daily) at 3 and 12 months of treatment would reverse changes observed in the airway microvasculature and the angiogenic factors VEGF and Angl, as well as VEGF receptors VEGFRI and VEGFR2, in patients with mild to moderate steroid-free asthma. In this "test of concept" study we hypothesised that treatment with high dose ICS would significantly reduce subepithelial vascularity and associated growth factors, especially VEGF.

\section{METHODS}

Thirty-five patients with mild to moderate symptomatic atopic asthma were recruited. All fulfilled ATS criteria for asthma which had been well documented for at least 12 months. Their mean (SD) age was 39 (13) years (range 20-70). None were current smokers; 16 were ex-smokers who had given up a median of 16 years (interquartile range 6-22 years) before recruitment. There was no clinical or physiological evidence of

Table 1 Mean vessel, growth factor and receptor staining levels per $\mathrm{mm}^{2}$ at baseline

\begin{tabular}{llll}
\hline & Placebo & ICS & Significance \\
\hline Vessels & $374(29)$ & $466(37)$ & NS \\
VEGF & $565(76)$ & $564(81)$ & NS \\
VEGFR1 & $361(29)$ & $324(19)$ & NS \\
VEGFR2 & $514(114)$ & $443(44)$ & NS \\
Ang 1 & $471(49)$ & $532(42)$ & NS \\
\hline
\end{tabular}

ICS, inhaled corticosteroid; VEGF, vascular endothelial growth factor; VEGFR1, VEGFR2, vascular endothelial growth factor receptors 1 and 2; An 1, angiopoietin-1.

There were no significant differences between the two groups. smoking-related disease in any participant in the study. Subjects were using only inhaled salbutamol for relief of symptoms and no "preventer" medication: in particular no subject had received treatment with regular ICS or inhaled long acting $\beta_{2}$ agonist for at least 12 months before the study. Only one subject had received a short course of oral corticosteroid treatment previously, but not within the last 3 months. Full details of the clinical status and study parameters can be found in our recently published work. ${ }^{15}$

Following recruitment there was a 2 week run-in period during which the subjects were continued on salbutamol alone as needed for symptomatic relief. Following this, bronchoscopic examination was performed with a $180 \mathrm{ml}$ standardised bronchoalveolar lavage (BAL) and up to six airway biopsy specimens were taken.

The subjects were then randomised to one of the following treatment groups: FP $750 \mu \mathrm{g}$ twice daily via metered dose inhaler or placebo (twice daily) via an identical metered dose inhaler. Of the 34 recruited patients, only one withdrew from the active treatment group. Bronchoscopy and airway sampling were again performed after 3 and 12 months of treatment. We were unable to obtain sufficient samples at 12 months to give statistically relevant data as only seven subjects remained in the study, which was insufficient for meaningful statistical power. However, qualitative examination of the data strongly suggested that there were no further changes between the 3 and 12 month periods in vessels or growth factors, so our analysis has been restricted to the 3 month data.

For bronchoscopic examination, subjects were premedicated with intravenous $0.4 \mathrm{mg}$ atropine and $5-15 \mathrm{mg}$ midazolam. Nebulised salbutamol ( $5 \mathrm{mg}$ ) was also given 15 minutes before bronchoscopic examination. Lignocaine (4\%) was applied topically to the nose, pharynx, and larynx and $2 \%$ lignocaine below the cords in $2 \mathrm{ml}$ aliquots as required up to a maximum dose of $6 \mathrm{ml}$. Subjects were monitored using pulse oximetry and administered oxygen during the procedure. All airway pathological end points were read blind to any clinical or treatment information, and admixed with normal control material taken from other concurrent studies (data not shown).

The study was approved by the institutional ethics committee and all subjects gave written informed consent.

\section{Vessel staining and quantification}

Biopsy specimens were embedded into the hydrophilic polymer glycol methacrylate (GMA). Coded blocks were cut on a semithin resin microtome (Leica RM 2310, Leica Microsystems, Nussloch, Germany) using glass triangular knives. The $2 \mu \mathrm{m}$ sections were then floated on water and adhered to silanised slides. Immunohistochemical staining was performed using anti-collagen type IV antibody to outline the endothelial basement membrane of airway vasculature. Total numbers of vessels per unit area were counted in quantifiable lamina propria to a depth of $150 \mu \mathrm{m}$ below the epithelial basement membrane. Angiogenic "sprouts" (fig 1) were simultaneously counted and the total number of sprouts per unit area and the mean number of sprouts per vessel were recorded.

\section{Growth factor detection}

Biopsies were fixed in $10 \%$ formalin for 2 hours and then embedded in paraffin wax. Blocks were cut on a Leica RM2155 microtome (Leica Microsystems) at $3 \mu \mathrm{m}$ thickness and sections were adhered to silanised glass slides. Extensive preliminary studies showed that GMA was poor for these immunostaining end points. Staining was performed with monoclonal antibodies for VEGF (R\&D Systems, Minneapolis, Minnesota, USA), VEGFRl, VEGFR2 (Santa Cruz Biotech, Santa Cruz, California, USA) and Angl (Jomar Diagnostics, 


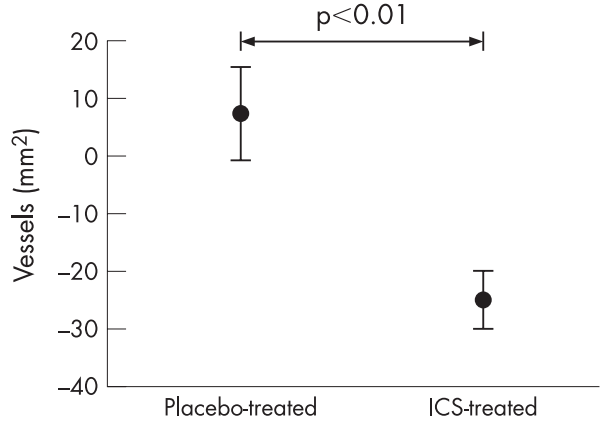

Figure 2 Percentage change in stained vessels per $\mathrm{mm}^{2}$ lamina propria in airway biopsies relative to placebo after treatment with inhaled corticosteroid (ICS). Bars are standard errors.

Stepney, South Australia, Australia) staining. For both types of preparation an ABC avidin-HRP kit (Vectastain Elite ABC Kit, VECTA Laboratories, Burlingame, California, USA) was used for secondary antibody binding and colour resolution. Growth factor staining was predominantly associated with vessels and quantified as stained vessels per unit area. The concentration of VEGF in BAL supernatant was measured using the QuantiGlo human VEGF immunoassay (R\&D Systems, USA).

Quantitative analysis of the biopsy sections was performed with a Leika DM LB microscope (Leica Microsystems), DageMTI DC200 one-chip video camera and Image Pro V4.l (Media Cybernetics, Sliver Spring, Maryland, USA) software.

\section{Analysis of data}

Differences between treatment groups were analysed using the Student's $t$ test either on the raw data (if parametric) or on $\log _{10}$ transformed data (if non-parametric). All data were expressed as means (standard error of the mean (SEM)). Differences were considered significant at $\mathrm{p}<0.05$. All data were compared with baseline values-that is, as the percentage change from baseline in either the placebo or treatment groups after treatment. These differences were used to compare the placebo and treatment groups using standard $t$ tests.

\section{RESULTS}

\section{Baseline data}

To reduce statistical error after treatment, the placebo and ICS treated groups were examined at baseline for statistical differences in vessel numbers, VEGF staining and receptor levels and Angl levels. No differences were found between the groups before treatment. These data are summarised in table 1 .

\section{Vessel numbers}

After 3 months of treatment with ICS the number of vessels/ $\mathrm{mm}^{2}$ in airway biopsy specimens was significantly reduced (ICS group: -122 (31) vessels $/ \mathrm{mm}^{2}$, placebo group: +10 (41) vessels/ $\left.\mathrm{mm}^{2} ; \mathrm{p}=0.002\right)$. In percentage terms this equated to a decrease of $25(5) \%$ in the ICS group $(\mathrm{p}<0.01)$ compared with an increase of $7(8) \%$ in the placebo group (fig 2).

\section{VEGF staining and levels}

Treatment with ICS had a significant effect on VEGF staining in biopsy sections. In the ICS group VEGF staining was reduced by -238 (141) stained vessels $/ \mathrm{mm}^{2}(-45$ (7)\%) compared with a significantly smaller reduction in the placebo group of -119 (128) stained vessels $/ \mathrm{mm}^{2}(-23 \quad(9) \% ; \mathrm{p}=0.04$, fig 3$)$. However, concentrations of VEGF in asthmatic BAL supernatant specimens were unchanged after 3 months of treatment with either placebo or ICS (fig 4).

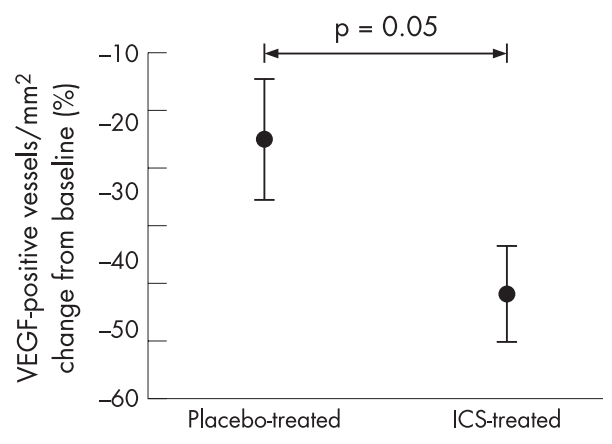

Figure 3 Percentage change in vascular endothelial growth factor (VEGF) stained vessels per $\mathrm{mm}^{2}$ of lamina propria in airway biopsies relative to placebo after treatment with inhaled corticosteroid (ICS). Bars are standard errors.

\section{VEGF receptor and Ang 1 staining}

No significant absolute changes were observed in immunostaining for VEGFR1 or VEGFR2 in bronchial biopsy specimens from placebo or ICS treated subjects (figs 5 and 6). Similarly, the absolute number of positively stained Angl vessels $/ \mathrm{mm}^{2}$ did not change significantly at 3 months in either group (fig 7).

Examining the relative staining for growth factors and receptors as a ratio of the number of vessels, we found that the percentage of total vessels $/ \mathrm{mm}^{2}$ stained with VEGF was lower with ICS ( $p=0.04$ within group), but not relative to coincidental changes in placebo. The percentage of vessels stained with VEGFR2 showed a trend towards an increase after treatment $(p=0.09)$ relative to placebo, as did Angl/vessel $(p=0.07)$. The percentage of vessels stained with VEGFRl was somewhat increased, but similar relative to placebo (table 2 ).

Treatment with ICS significantly reduced the number of sprouts per vessel from $4.72(0.61)$ at baseline to $3.42(0.68)$ after 3 months of treatment $(\mathrm{p}<0.01)$; again, no significant changes were observed in the placebo group. Analysis of the percentage changes between both treatment groups showed that ICS significantly reduced the number of sprouts per vessel from baseline to 3 months compared with placebo (ICS -29.1 $(5.8) \%$ vs placebo $+23.6(18.7) \%$; $p=0.02$, fig 8$)$.

\section{DISCUSSION}

Inhaled corticosteroids are the most potent anti-inflammatory agents available for the treatment of asthma. ${ }^{16}$ Their total effect

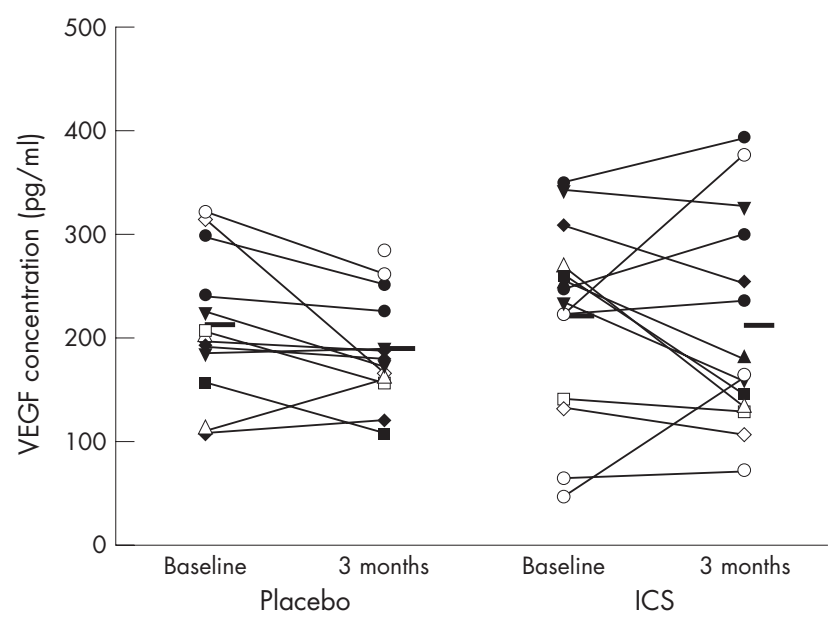

Figure 4 Vascular endothelial growth factor (VEGF) levels in bronchoalveolar lavage supernatant before and after 3 months of treatment with inhaled corticosteroid (ICS) or placebo. 


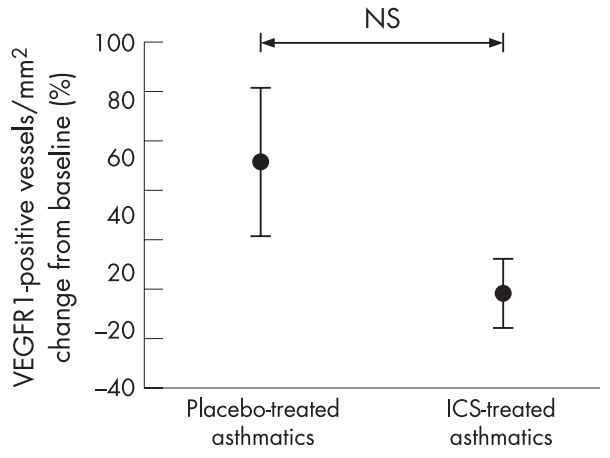

Figure 5 Percentage change in vascular endothelial growth factor receptor 1 (VEGFR 1) stained vessels per $\mathrm{mm}^{2}$ lamina propria in airway biopsies relative to placebo after treatment with inhaled corticosteroid (ICS). Bars are standard errors.

is known to be related to many factors including the attenuation of inflammatory cell numbers, function and activation; stabilisation of vascular leakage; a decrease in mucus production; and an increase in $\beta$-adrenergic response. ${ }^{17}$ In addition, we have previously shown a decrease in airway scar collagen deposition, but only after a 12 month period of treatment. ${ }^{18}$ In this study we have shown that 3 months of treatment with high dose FP ( $750 \mu$ g twice daily) was able to reduce the total number of vessels within the lamina propria of airways in ICS treated asthmatic patients. Twelve months of treatment had no further effect.

Concomitant with the reduction in vessel numbers during the 3 month initial treatment period, ICS also decreased VEGF immunostaining, but proportionately to the decrease in vessel numbers. In contrast, no ICS-related changes were observed in BAL fluid concentrations of VEGF. There was no absolute change in VEGF receptors (VEGFRl or VEGFR2) or Angl immunostaining but, in relation to the change in vessel numbers, expressions of VEGFR2 and Angl were slightly higher after treatment. We also observed a proportionate decrease in the numbers of angiogenic sprout-like structures within the vascular wall of vessels after ICS treatment, as further evidence of ICS-induced downregulation of vascular remodelling.

The relationship between ICS dosage and subepithelial vascularity has been examined previously by our group. ${ }^{11}$ We found an inverse relationship between ICS dosage and airway wall vascularity in patients with asthma of comparable clinical disease activity, with higher dose beclomethasone dipropionate treatment ( $\geqslant 800 \mu \mathrm{g} /$ day) being associated with reduced vessel

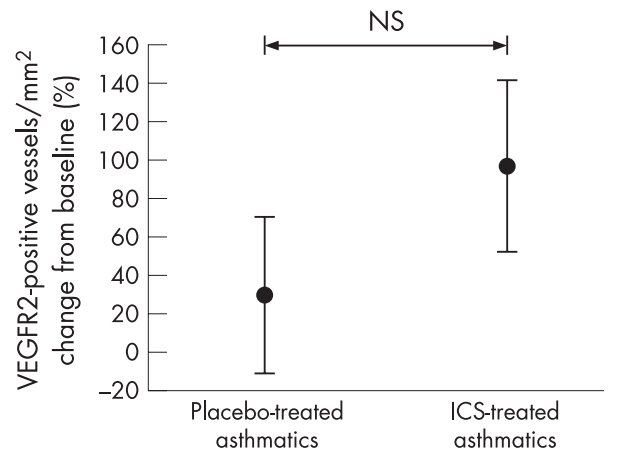

Figure 6 Percentage change in vascular endothelial growth factor receptor 2 (VEGFR2) stained vessels per $\mathrm{mm}^{2}$ lamina propria in airway biopsies relative to placebo after treatment with inhaled corticosteroid (ICS). Bars are standard errors.

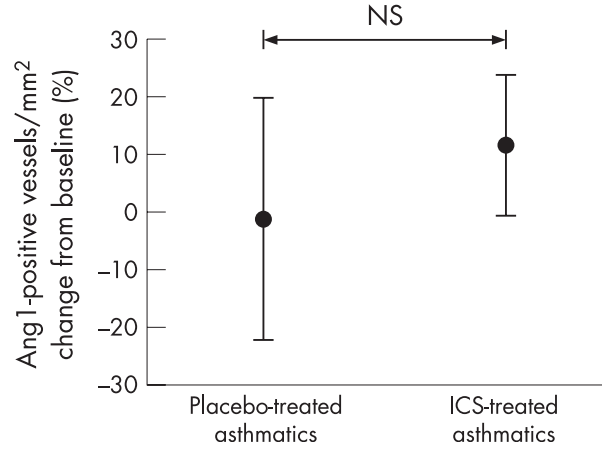

Figure 7 Percentage change in angiopoietin-1 (Ang 1) stained vessels per $\mathrm{mm}^{2}$ of lamina propria in airway biopsies relative to placebo after treatment with inhaled corticosteroid (ICS). Bars are standard errors.

numbers. In addition, in many of those receiving high dose ICS, the vessel numbers fell within the normal range of healthy controls, a finding also reported by others after 6 weeks of high dose FP treatment. ${ }^{18}$ Together, these findings suggest that higher doses of ICS may be required to consistently reverse airway hypervascularity in asthma.

This study supports these previous findings and further examines the contribution of the angiogenic factors VEGF and Angl, as well as VEGFRl and VEGFR2, and the potential role that they may have in the microvascular remodelling process in asthma. Our data suggest that VEGF expression within the airway wall is responsive to ICS, and this may be the means by which ICS causes regression of hypervascularity in the airway wall. While VEGF vessel staining was reduced in relation to the absolute number of vessels after treatment, no parallel changes were observed in Angl, VEGFRl or VEGFR2 immunostaining. In examining the ratios of these factors per vessel, we observed an indication that VEGFR2 and Angl expression could be proportionately increased after treatment. Although speculative, given the data, relative increases in VEGFR2, the active VEGF receptor, and Angl, a vascular stabilising factor, might suggest that conditions in the airways after ICS treatment are less angiogenic.

VEGF concentrations did not change in the BAL fluid in ICStreated asthmatics. This disparity with VEGF staining may be explained by regional differences in the airway distribution from which the specimens were obtained: endobronchial biopsy specimens were obtained from proximal airways (generations 3-4) whereas BAL fluid specimens may better sample the smaller peripheral airways. Regional differences in FP deposition between large and small airways due to the size of the FP particles may account for the observed disparity in the results.

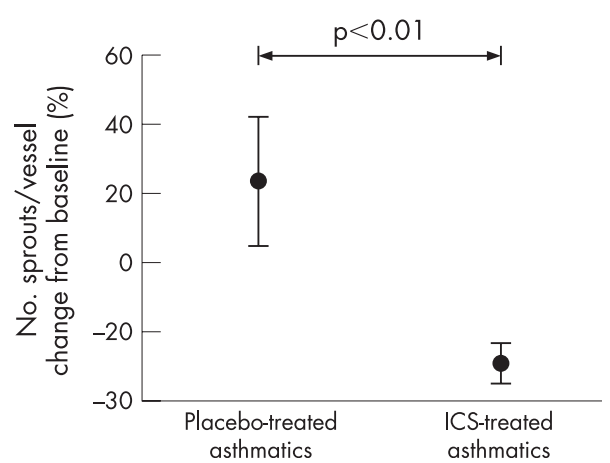

Figure 8 Percentage change in number of vascular sprouts per vessel relative to placebo after treatment with ICS. Bars are standard errors. 
Table 2 Ratio of stained growth factor to vessel numbers in airway biopsy specimens before and after treatment with ICS or placebo

\begin{tabular}{|c|c|c|c|c|c|}
\hline & \multicolumn{2}{|c|}{ Mean vessel staining (pre-ICS) } & \multicolumn{2}{|c|}{ Mean vessel staining (post-ICS) } & \multirow{2}{*}{$\begin{array}{l}\text { Significance of ICS } \\
\text { treatment change vs } \\
\text { placebo }\end{array}$} \\
\hline & ICS (\%) & Placebo (\%) & ICS (\%) & Placebo $(\%)$ & \\
\hline VEGF & $100(12)$ & $100(17)$ & $75(9)$ & $85(13)$ & NS \\
\hline VEGFR 1 & $65(8)$ & 70 (11) & 100 (10) & $86(9)$ & NS \\
\hline VEGFR2 & $42(9)$ & $55(13)$ & 100 (14) & $67(8)$ & NS $(p=0.09)$ \\
\hline Angl & $68(3)$ & $74(7)$ & $100(5)$ & $72(8)$ & NS $(p=0.07)$ \\
\hline
\end{tabular}

ICS, inhaled corticosteroid; VEGF, vascular endothelial growth factor; VEGFR1, VEGFR2, vascular endothelial growth factor receptors 1 and 2; Anl, angiopoietin-1.

VEGF staining at baseline was normalised to $100 \%$ and receptor staining is given as a percentage of the respective vessels counted.

Nevertheless, BAL fluid levels of VEGF were strongly associated with subepithelial vascularity at baseline. ${ }^{15}$ After 3 months of ICS treatment, however, this relationship was no longer evident (data not shown). This might suggest that VEGF levels in BAL fluid reflect some clearance mechanism by which VEGF is moved out into the airway lumen after ICS treatment, away from angiogenic receptors. Such "luminal clearance" mechanisms for airway pro-inflammatory cytokines have previously been suggested. ${ }^{19}$

ICS-induced reduction of the subepithelial vasculature within the airway wall may have considerable functional implications, given that the microvascular bed is a substantial component of the airways ( $\sim 20 \%$ of the area of the airway wall). Even small alterations to the thickness of the inner airway wall can contribute to enhanced airway narrowing in asthma. ${ }^{12}$ Despite this, the reduction in subepithelial vascularity after ICS treatment in this study was not directly proportional to clinical or physiological improvement in this study (regression not shown), nor in several other studies. ${ }^{10}{ }^{18}{ }^{20} \mathrm{We}$ and others have found such an association cross-sectionally in asthma and have shown significant inverse correlations between subepithelial vascularity and $\mathrm{FEV}_{1}$ and BHR, suggesting that the degree of vascularity and potential capacity for engorgement of the airway wall may play a role in diminished lung function and the pathogenesis of BHR in asthma. ${ }^{11}{ }^{21}$ Thus, the acute physiological state of the airway may be an important factor in demonstrating the importance of angiogenesis - that is, whether the vessels themselves are dilated and/or engorged as part of an acute inflammatory challenge, and whether the airway muscle deep to the vascular plexus is being constricted. Lack of a relationship between vessels and other manifestations of asthma under resting and well controlled conditions may be less meaningful.

Further evidence of the modulation of airway vascularity by ICS is demonstrated in the observed reduction in vascular "sprouts" after ICS treatment. In a previous paper ${ }^{15}$ we showed differences between control and asthmatic subjects in these vascular structures but were unable to determine their exact nature. We have now shown that they are sensitive to ICS treatment and are greatly reduced in an environment where total vessel numbers are decreasing with treatment. While it is possible that these sprouts are not angiogenic structures per se, there can be little doubt that they are at least an indicator of vascular remodelling.

This study supports the hypothesis that high dose ICS downregulates microvascular angiogenic remodelling in asthma. The observed reduction in subepithelial vascularity following 3 months of treatment with high dose FP ( $750 \mu$ twice daily) was associated with a concomitant and proportionate reduction in VEGF-positive vessels per unit area, and a reduction in angiogenic sprouts per vessel. Further, the relationships between VEGF, both stained within airway vessels and in the BAL fluid, were quite different after ICS treatment compared with baseline.
These changes, as well as the relative changes to VEGF receptors and Angl staining, suggest subtle re-setting and probable stabilisation of angiogenic control mechanisms in the airway wall with ICS treatment.

\section{Authors' affiliations}

B N Feltis, D W Reid, E H Walters, Cardio-Respiratory Research Group, University of Tasmania, Hobart, Australia

D Wignarajah, Department of Medicine, Monash University, Melbourne, Australia

C Ward, Applied Immunobiology and Transplantation Group, University of Newcastle upon Tyne, UK

R Harding, Department of Anatomy and Cell Biology, Monash University, Australia

Competing interests: None

\section{REFERENCES}

1 Rhen T, Cidlowski JA. Antiinflammatory action of glucocorticoids-new mechanisms for old drugs. N Engl J Med 2005;353:1711-23.

2 Haahtela T, Jarvinen M, Kava T, et al. Comparison of a beta 2-agonist, terbutaline, with an inhaled corticosteroid, budesonide, in newly detected asthma. N Engl J Med 1991;325:388-92.

3 Diukanovic R, Wilson JW, Britten KM, et al. Effect of an inhaled corticosteroid on airway inflammation and symptoms in asthma. Am Rev Respir Dis 1992;145:669-74.

4 Derendorf H. Hochhaus G, Meibohm B, et al. Pharmacokinetics and pharmacodynamics of inhaled corticosteroids. J Allergy Clin Immunol 1998; 101(4 Pt 2):S440-6.

5 Ward C, Pais M, Bish R, et al. Airway inflammation, basement membrane thickening and bronchial hyperresponsiveness in asthma. Thorax 2002:57:309-16.

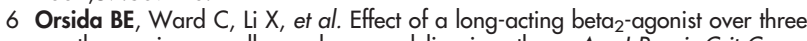
months on airway wall vascular remodeling in asthma. Am J Respir Crit Care Med 2001; 164:117-21.

7 Chetta A, Zanini A, Foresi A, et al. Vascular endothelial growth factor upregulation and bronchial wall remodelling in asthma. Clin Exp Allergy 2005;35: 1437-42

8 Hashimoto $M$, Tanaka H, Abe S. Quantitative analysis of bronchial wall vascularity in the medium and small airways of patients with asthma and COPD. Chest 2005; 127:965-72.

9 Hoshino M, Takahashi M, Aoike N. Expression of vascular endothelial growth factor, basic fibroblast growth factor, and angiogenin immunoreactivity in asthmatic airways and its relationship to angiogenesis. J Allergy Clin Immunol 2001;107:295-301.

10 Li X, Wilson JW. Increased vascularity of the bronchial mucosa in mild asthma. Am J Respir Crit Care Med 1997;156:229-33.

11 Orsida BE, Li X, Hickey B, et al. Vascularity in asthmatic airways: relation to inhaled steroid dose. Thorax 1999:54:289-95.

12 Moreno RH. Hogg JC. Pare PD. Mechanics of airway narrowing. Am Rev Respir Dis 1986;133:1171-80.

13 Maisonpierre PC, Suri C, Jones PF, et al. Angiopoietin-2, a natural antagonist for Tie2 that disrupts in vivo angiogenesis. Science 1997;277:55-60.

14 Suri C, McClain J, Thurston G, et al. Increased vascularization in mice overexpressing angiopoietin-1. Science 1998;282:468-71.

15 Feltis BN, Wignarajah D, Zheng L, et al. Increased vascular endothelial growth factor and receptors: relationship to angiogenesis in asthma. Am J Respir Crit Care Med 2006;173:1201-7.

16 Barnes PJ. Inhaled glucocorticoids: new developments relevant to updating of the asthma management guidelines. Respir Med 1996;90:379-84.

17 Lemanske RF Jr, Busse WW. 6. Asthma. J Allergy Clin Immunol 2003;111(2 Suppl):S502-19. 
18 Chetta A, Zanini A, Foresi A, et al. Vascular component of airway remodeling in asthma is reduced by high dose of fluticasone. Am J Respir Crit Care Med 2003;167:751-7.

19 Feltis BN, Reid DW, Ward C, et al. BAL eotaxin and IL-5 in asthma, and the effects of inhaled corticosteroid and beta $_{2}$ agonist. Respirology 2004;9:507-13.
20 Orsida BE, Ward C, Li X, et al. Effect of a long-acting beta2-agonist over three months on airway wall vascular remodeling in asthma. Am J Respir Crit Care Med 2001; 164:117-21

21 Hoshino M, Takahashi M, Takai Y, et al. Aoike N. Inhaled corticosteroids decrease vascularity of the bronchial mucosa in patients with asthma. Clin Exp Allergy $2001 ; 31: 722-30$.

\section{bmjupdates+}

bmjupdates+ is a unique and free alerting service, designed to keep you up to date with the medical literature that is truly important to your practice.

bmjupdates+ will alert you to important new research and will provide you with the best new evidence concerning important advances in health care, tailored to your medical interests and time demands.

Where does the information come from?

bmjupdates+applies an expert critical appraisal filter to over 100 top medical journals

A panel of over 2000 physicians find the few 'must read' studies for each area of clinical interest

Sign up to receive your tailored email alerts, searching access and more...

www.bmjupdates.com 